\title{
Modeling of selective laser sintering/selective laser melting
}

\author{
Connor West, Xuan Wang* \\ Industrial and Manufacturing Engineering Department, California Polytechnic State University, San \\ Luis Obispo, CA 93407
}

\begin{abstract}
Selective laser sintering and selective laser melting are powder based additive manufacturing (AM) process that can rapidly manufacture parts with comparable mechanical properties to conventional manufacturing methods directly from digital files. However, the processing recipe development and design optimization of AM parts are often based on trial and error which erodes the benefit of AM. Modeling is a powerful tool to enable faster development cycle by significantly reducing the experimental efforts. In this paper we discussed the current status of selective laser sintering/melting modeling, in which the laser and powder interaction was studied to understand and predict the process and the properties of fabricated parts. A review of the current approach as well as future directions are presented.
\end{abstract}

Keywords: Powder bed fusion, modeling, selective laser melting, selective laser sintering

\section{INTRODUCTION}

Powder bed fusion (PBF) additive manufacturing (AM) is the most popular manufacturing process for directly manufacturing of metallic parts. Processes using laser as the power source, including selective laser sintering (SLS) and selective laser melting (SLM), which utilize a high powered laser to "selectively" fuse material powder according to the CAD model, layer by layer, into a three dimensional part, is the predominant process in PBF. SLS technology has been emerging as a manufacturing process due to its ability to quickly produce complex geometry features for prototyping as well as high end production parts. SLS parts are built upon a powder bed which supports the subsequent layers allowing for internal channels and overhanging structures to be built without support material. A special type of SLS that is specific to metal alloys is called Direct Metal Laser Sintering (DMLS). SLM is a very similar process to SLS, but in SLM the material is fully melted into one homogeneous part. The complete melting of the powder allows for near full density parts to be achieved. SLM can be more difficult to control than SLS due to issues of incomplete powder melting and material phase changes occurring at the higher temperatures [1]. In general, SLS and SLM can be used with metals, plastics, nylon, glass, and ceramics. Since smaller wavelengths are more easily absorbed by metals, fiber lasers (1064 $\mathrm{nm}$ ) are typically used in SLS and SLM. The wide array of materials that can be used in these processes allow for customization over the porosity and mechanical properties, for instance, in the automotive and aerospace industries, where they are able to manufacture lightweight parts to save money and improve performance. Other benefits include custom control over part density by varying process parameters, and the freedom to construct parts without restriction on internal features. As a very powerful manufacturing technique, SLS/SLM are very complicated processes due to the various thermo-mechanical phenomena occurring. The large amount of heat output from the laser and the repeated temperature cycles from the layer by layer approach results in a nonlinear thermal field within the part. As a result, several defects arise such as the balling effect, residual stresses, incomplete melting, and part distortion [2].

The interaction between laser and powder materials will determine the binding mechanisms. There are four binding mechanisms in SLS/SLM: Solid State Sintering (SSS), Liquid Phase Sintering (LPS), partial melting and fully melting [3]. Depending on the laser parameters, such as power, spot size, scanning speed, hatching distance, and scanning strategy, different binding mechanisms occur during SLS/SLM. Under the irradiation of laser beam, the powders will start densification process, in which porosity, microstructure, and residual stress will evolve. The mechanical properties and geometries will also evolve accordingly on the macroscopic level. Figure 1 illustrates the schematics of inputs and outputs of SLS/SLM process.

To the end users, mechanical properties and geometries of fabricated parts are the most critical. Thus it is desired to have predictable mechanical properties and geometries in order to have confidence in the production. Currently, the inability of AM technology to guarantee material properties for a given process is inhibiting its industry adoption because many companies do not have confidence that manufactured parts will have the mechanical properties required to meet specific

*xwang12@ calpoly.edu; phone: 805-756-2137

Laser 3D Manufacturing IV, edited by Bo Gu, Henry Helvajian, Alberto Piqué, Corey M. Dunsky,

Jian Liu, Proceedings of SPIE Vol. 10095, $1009506 \cdot$ @ 2017 SPIE

CCC code: $0277-786 \mathrm{X} / 17 / \$ 18 \cdot$ doi: $10.1117 / 12.2256539$ 
application needs [4]. In subtractive processes, as long as materials and machining process are fixed, the mechanical properties and geometries are fairly predictable, no matter what equipment vendor or model is selected. However, this is not the case in SLS/SLM. Using the same materials on different machines even with same recipe will end up with very different mechanical properties. In AM, its technological capability and used materials are absolutely dependent; there is no combinatorial freedom at all. For the first time in manufacturing, a system inherently includes dedicated component materials [5]. For example, cracks, long elongated pores and intermediate pores have been observed in the sintered alumina powders because of the un-optimized SLS parameters [6]. For this reason, every commercial SLS/SLM equipment are tuned to proprietary powder materials; only using the exact combination of materials, equipment, and process window can ensure successful build. For dense metal parts with minimum surface irregularities and geometric distortion, several material and processing issues must be fine-tuned simultaneously [7]. For example, EOS Gmbh developed proprietary powder mixtures to enable near net shaping. The shrinkage that takes place during sintering is balanced by the expansion of the powder particles during sintering. The reason for this exceptional behavior lies in the tailored multi-component powder systems that compensates for the natural shrinkage tendency of liquid phase sintering by means of an inter-diffusional swelling phenomenon [8].

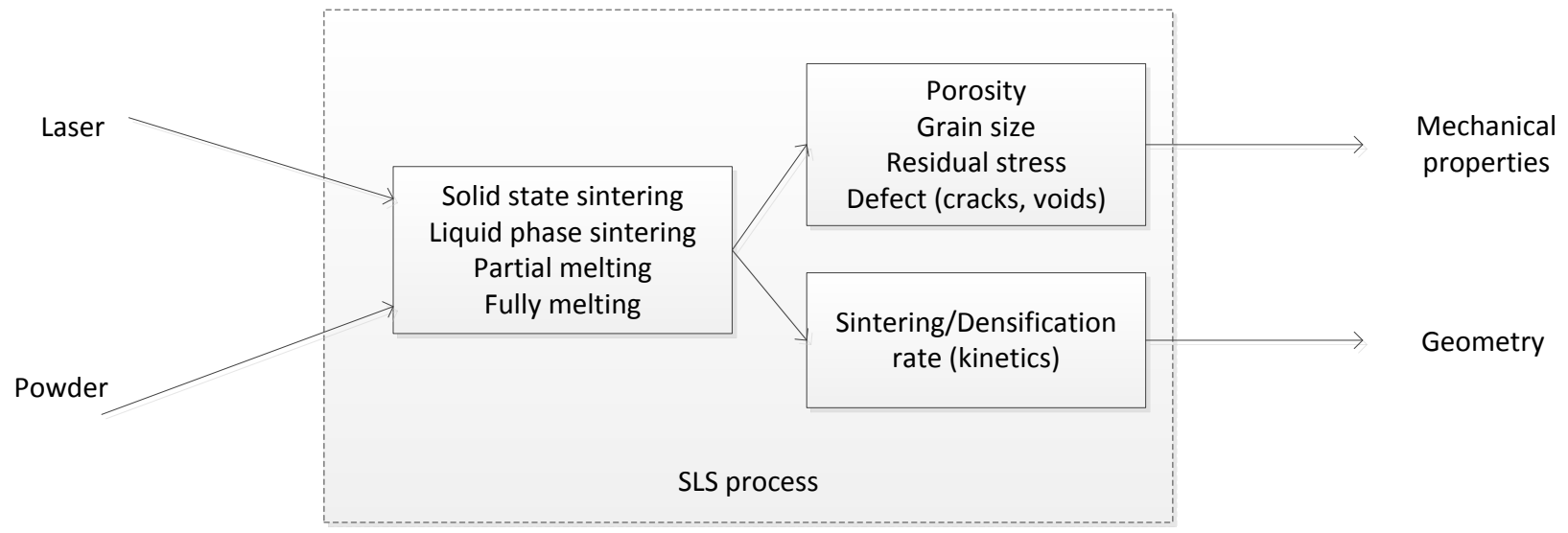

Figure 1 the schematics of relationship of inputs and outputs of SLS/SLM process

Even using same material and equipment, if there are slightly changes in design, one cannot guarantee to get the part with desired mechanical properties and geometries. Several iterations may be required to find out the optimal processing window. This is one of the major barriers to prevent the broader adoption of SLS as a truly dependable manufacturing process. For example, same design with different build orientations can cause significant differences in mechanical properties and failure mode because of the anisotropy of SLS process [9]. This also contributes to the high manufacturing cost of SLS parts because equipment, processing recipe and material were controlled by a limited number of companies. The users don't have the freedom to choose materials; if they purchased equipment from a vendor, they must also purchase materials from same vendor to ensure build quality. During the NSF workshop in 2009 on AM roadmap, one of the important directions identified is composable simulation capabilities for primitive shapes, materials, and material compositions. Multi-scale modeling and inverse design methodologies to assist in navigating complex process-structure-property relationships, and improved finite element analysis software that can make use of such capabilities are extremely critical [10].

Multi-scale, multi-physics modeling, and experimental validation are imperative to understand the laser's interaction with powder materials to determine the binding mechanisms accordingly. Modeling on microscopic and macroscopic level can be conducted depending on different binding mechanisms. On microscopic level, porosity evolution, microstructure evolution and particle coalescence, could be modeled to predict final mechanical properties. On the macroscopic level, constitutive modeling is needed to understand the kinetics of sintering and densification process; from which the geometry and properties can be predicted. However, there is no generalized approach with above mentioned capabilities. Only limited research has been conducted on specific scenarios which limited the usage. The overview of the current status in the field of SLS/SLM modeling and experiment will be discussed in the next section. 


\section{THERMAL MODELING}

In the Additive Manufacturing Roadmap Workshop organized by NSF in 2009, developing predictive process-structureproperty relationships integrated with $\mathrm{CAD} / \mathrm{E} / \mathrm{M}$ tools has been identified as a key focus area [10]. The nature of close coupling of thermal, chemical, material, and environment in SLM makes a single model very challenging. Slicing the problem into multi scales and multi physics is the primary focus at the current stage.

\subsection{Gaussian laser distribution}

As the laser beam traces the powder layer, a molten melting pool is formed in which the fusing of the powder occurs. Two of the most important process parameters that control the dimensions of the molten pool as well as the quality of the particle fusing are the laser power and laser scanning speed [11]. One critical problem that arises from inadequate process parameters is a phenomenon called the balling effect. The exact cause of the balling effect is unknown but the molten pool has a tendency to shrink releasing surface energy, allowing the molten pool to break into separate balls. The balling effect is problematic because it increases the surface roughness and degrades mechanical properties of the part due to low quality fusing [12]. The size and dimensions of the molten pool are known to be a determining factor as to whether the balling effect will occur as it has been observed to occur when the length to diameter ratio of the molten pool becomes greater than " $\pi$ " [2]. As a result, the laser intensity and scanning speed are two of the most important process parameters to control part integrity. Other contributing factors to balling initiation is the oxygen content in the atmosphere, powder layer thickness, and the type of powdered material.

The most widely accepted laser model is the Gaussian beam model. This model assumes that the beam has a Gaussian irradiance profile where the maximum intensity is located at the center of the beam and is symmetrically distributed from the beam axis. The fundamental equation for the beam irradiance is described in literature [13]. Fu and Guo [2] state that a shape factor of 2 is typically used for the Gaussian heat flux distribution $(B=2)$. The absorptivity of the powder material is typically taken as the absorptivity for the bulk material $[1,14]$. The majority of past simulations have used a surface heat flux due since the powder layer is typically very thin. Alternatively, a few studies have used a 3D volumetric heat flux instead of the 2D surface heat flux. The most notable use of a volumetric heat flux is the study by Hodge [15]. They rationalize that it is possible that the heating of a powder that is not closely packed may occur within the bulk of the material. As a result, the heating is not only at the surface and thus a volumetric heat flux was used.

\subsection{Temperature dependent material properties}

The thermo-mechanical properties between a solid bulk body and a powder layer of the same material differ. Additionally, since the SLS/SLM processes involve a material state change some properties will vary with temperature. Some of the key material properties needed to complete 3D heat conduction analysis include the density, thermal conductivity, and the specific heat of the powder. Two of the most common materials used in SLS/SLM applications are Ti-6Al-4V and 316L stainless steel. The density of the powder layer is much smaller than the bulk material and as a result has a much higher absorptivity. Sih et. al. proposed an effective model to estimate the thermal conductivity of ceramic powders and compared with experiment [16]. Hussein [13] state that the absorptivity of the powder material is a function of the reflectivity of the material. For example, the absorptivity of 0.3 and the reflectivity of 0.7 were used to calculate the absorptivity for 316L stainless steel [4]. As a comparison Fu [2] used the absorption coefficient for pure titanium powder with the value of 0.77 ). Also, the thermal conductivity of the powder layer is much smaller than that of the bulk material. This is due to the larger porosity within the powder layer which results in a smaller contact area. In addition, gas fills the voids in the porous powder which has a much lower thermal conductivity. Thus the compactness of the powder layer plays a critical role in analyzing the heat conduction during SLS/SLM. Packing density can be modeled as a function of particle size and shape distribution and has been described by several models [17].

\subsection{Layer addition}

One important aspect of modeling is to be able to account for the addition of multiple layers. Roberts [14] focused on analysis of the temperature field during laser melting through simulation of the "element birth and death" method. This method is able to model layer addition by activating new elements or layers at specific time intervals. This enables the ability to model the time delay and cooling effect of rolling a new layer of powder before the next laser scan. Only once the elements are activated are they included in the overall stiffness matrix. Through this method, it was concluded that 
successive laser scanning over additional layers will affect the temperature distribution of previous layers. The reheating of layers creates secondary peaks in temperature as well as a small build-up of temperature in the part and substrate.

Several research studies on different SLS and SLM process characteristics have been conducted in the past. The most common modeling technique involves use of the finite element analysis method (FEA) using either Abaqus or ANSYS software. The most relevant studies conducted include a focus on modeling layer build up and a moving heat source. The summary of literature is listed in Table 1.

Table 1 Summary of previous research studies involving modeling of SLS

\begin{tabular}{|l|l|l|}
\hline Research Focus & Material & Source \\
\hline $\begin{array}{l}\text { Melting pool shape and dimensions, Temperature gradient and } \\
\text { thermal history, Effect of process parameters }\end{array}$ & Ti-6Al-4V & Fu and Guo (2014) \\
\hline Temperature history from the addition of layers & Ti-6Al-4V & Roberts et al. (2009) \\
\hline Temperature and stress fields in single layers built without-support & 316L Stainless Steel & Hussein et al. (2013) \\
\hline Residual stresses and deformations & 316 Stainless Steel & Jiang et al. (2002) \\
\hline Computational reduction model to decrease modeling time & IN718 & $\begin{array}{l}\text { Papadakis et al. } \\
\text { (2014) }\end{array}$ \\
\hline Effect of process parameters on temperature evolution & 316L Stainless Steel & Contuzzi et al. (2011) \\
\hline Effect of process parameters on tensile properties & 304 Stainless Steel & Guan et al. (2013) \\
\hline Effect of volume shrinkage & Dental Porcelain & Dai and Shaw (2005) \\
\hline Balling "Effect" Behavior & $\begin{array}{l}\text { Stainless Steel, Nickel } \\
\text { Powder }\end{array}$ & Li et al. (2011) \\
\hline 2D analysis of thermal fields & Ti-6Al-4V & Teng, et al. (2015) \\
\hline
\end{tabular}

\section{MULTIPHYSICS MODELING}

A number of phenomenon can happen simultaneously during SLS/SLM, including heat transfer, phase change, intermetallic compound reaction, evaporation, solidification, grain growth, flow of molten pool, and equilibrium between solid/liquid/gas interface. To fully understand the process, Multiphysics modeling is required.

\subsection{Molten pool stability}

In SLM, the molten pool stability is key to influence the build quality and is responsible for a number of defects, such as balling. The surface tension, shape, as well as Marangoni flow can be impacted by the build environment of SLM chamber [17]. Gusarov et. al. [18] proposed a model for coupled radiation transfer and thermal diffusion is proposed, which provides a local temperature field. Comparison with experimental results showed balling effect which can be explained by the Plateau-Rayleigh capillary instability of the melt pool. Tang [19] used Rosenthanl's equation to predict the molten pool size and the resultant porosity. The continuity of the molten pool can be described using Plateau-Raleigh instability. Khairallah et. al. [20] developed a 3D meso scale powder model and studied the molten pool under different scanning strategy. To describe the interaction between the vapor and the molten pool, Masmoudi [21] used LennardJones potential was considered to describe interactions between the gas molecules (for estimation of the transport properties).

\subsection{Coupling with solidification}

Matsumoto et. al. [22] developed a model with solidification in which the heat caused by laser irradiation is given to the elements under the laser beam. Shrinkage due to solidification is assumed to result in only the change of the layer thickness. Ganeriwala [23] developed a powder model and thermal model incorporated with phase change, such as from a solid to a liquid, and possibly even to a gas. Li developed a multiscale model from meso scale to macro scale [24] to simulate the residual stress and distortion. Zhang investigated melting and shrinkage in a powder bed containing two powders with different melting points assuming constant heat flux using a 1D model [25]. Kolossov developed a thermal model to allow for the non-linear behavior of thermal conductivity and of specific heat due to temperature changes and phase transformations. The temperature evolution and the formation of the sintered part are simulated by a 3D finite element analysis based on continuous media theory [26]. Chung developed a 1-D heat transfer, melting, vaporization and re-solidification model describing the interaction of a scanning continuous-wave laser with a metal surface wherein the beam power is constant. The effects of processing parameters on process variables were investigated numerically by 
varying beam diameters, scan speeds and substrate temperatures for Nickel. Relations are derived for the times to initiate melting, to initiate vaporization, to reach maximum melting depth, for melting-resolidification, and for maximum melting and vaporization depths [27]. Olakanmi experimentally studied the role of processing parameters on the densification mechanism and microstructural evolution in laser sintered Al-12Si powder. It was established that both the densification mechanism and microstructural evolution in laser sintered Al-12Si powder were controlled by the specific laser energy input. Analysis of the cross-section of laser sintered microstructures of Al-12Si powders indicated that the tops of the grains in the previous layer are partially re-melted and then undergo epitaxial growth in the next layer where the heat affected zones (HAZ) grain boundaries and solidification grain boundaries (SGBs) are continuous along the fusion boundary [28]. These above-mentioned approaches normally assume a defined heat source, which in turn requires to postulate the powder absorption and the depth at which the absorption takes palaces [29]. However, not much work has been done to formulate the relationship between laser parameters, powder properties, microstructure evolution, and mechanical properties. There is a need to understand the effect of thermo-mechanical exposure on material microstructure and properties and to articulate the constitutive behavior of materials as a function of machine process variables [10]. Sun divided the conventional powder-based laser sintering into three model parts: the optical, the thermal and the sinter model [30]. Beam offset, inertia of scanning mirror and positioning errors in hatch generations are found to delude the shrinkage pattern [31]. Ganeriwala studied a thin layer of particle's SLS process using 2D FEM to simulate the temperature [23]. Paul developed a methodology to calculate the laser energy of a part manufactured in the SLS process and to correlate the energy to the part geometry, slice thickness and part orientation. The laser energy was correlated to the total area of sintering (TAS) by using a convex hull based approach [32]. Ragulya developed continual modeling on SLS based on viscous flow and non-linear sintering rheology [33]. The thermal gradients in the powder bed are then related to thermal stresses, enabling predication of warpage or distortion of the fabricated parts [34]. Dai used 3D thermo-mechanical finite element model including the effect of the powder-to-solid transition to investigate the transient temperature, transient stress, warpage and residual stress fields in processing of multiple material components [35].

\subsection{Density simulation}

Density is probably the single most important characteristic in SLS which will influence the final properties of parts significantly. Unfortunately, density prediction in SLS is challenging due to the following reasons: (1) the powder scale model and the effective media model on the macro scale must be linked. Such as scale magnitude will inevitably include a lot of factors and variations which makes the fidelity of the model questionable. (2) the computation power requires to understand the powder level is extremely intensive which is impractical in industrial applications. Simchi studied the effect of processing parameters on the densification of metal powders experimentally. Based on the empirical sintering rate data, a relationship was established between the densification of metal powders during Direct Metal Laser Sintering (DMLS) and the energy delivered to the powder bed by the laser beam. This relationship has been shown to be useful for metals with congruent melting point or alloys, which full melting/solidification approach is feasible mechanism of rapid particle bonding in DMLS process [34]. Simchi also studied the influence of particle size to the densification rate [36]. Want et. al. [37] developed a continuum model based on classical sintering theory [38] to simulate the densification of W-Ni-Fe.

\section{SUMMARY}

The ongoing research and the number of publications in modeling of SLS/SLM increase dramatically in the past decade, driven by the demand from industry to make the process predictable and repeatable comparable to the level of conventional manufacturing. Process qualification requires understanding on the physical level as well in practical applications, for example, to predict the defect and prevent them from happening. Due to the complexity of multi-physics involved in the process, multi-scale modeling is required. Current approach involves thermal modeling coupled with other phenomenon to simulate residual stress, part distortion, temperature field, and density. Meso scale to macro scale modeling has been successfully demonstrated. However, extensive validation with different geometries, materials, and machines are required to ensure the developed models are robust enough for practical applications. Moreover, the integration of simulation with existing CAD package to enable affordable computing is necessary. 


\section{REFERENCES}

[1] Teng, C., Pal, D., Gong, H., \& Stucker, B., “A Two Dimensional Analytical Evaluation of Thermal Fields during Metal Laser Sintering Processes,” Solid Freeform fabrication symposium, Univ. of Texas, 1145-1157 (2014).

[2] Fu, C. H., \& Guo, Y. B., "3-Dimensional Finite Element Modeling of Selective Laser Melting Ti-6Al-4V Alloy,” Solid Freeform fabrication symposium, Univ. of Texas, 1129-1144 (2014).

[3] J.P. Kruth, P Mercelis, J. Van Vaerenbergh, L Froyen, M Rombouts, "Binding mechanisms in selective laser sintering and selective laser melting," Rapid Prototyping Journal, 11 (1), 2-36 (2004).

[4] N. Guo, M. C. Leu, “Additive manufacturing: technology, applications and research needs,” Front. Mech. Eng., 8(3), 215-243 (2013).

[5] G. N. Levy, "The role and future of the laser technology in the additive manufacturing environment," Physics Procedia, 5, 65-80 (2010).

[6] J. Deckers, K. Shahzad, J. Vleugels and J.P. Kruth, Isostatic pressing assisted indirect selective laser sintering of alumina components, Rapid Prototyping Journal, 18 (5), 409-419 (2012).

[7] M. Agarwala, D. Bourell, J. Beaman, H. Marcus, J. Barlow, "Direct selective laser sintering of metals," Rapid Prototyping Journal, 1(1), 26-36 (1995).

[8] J. Hanninen, "Direct metal laser sintering," Advanced Materials \& Processes, 33 (2002)

[9] E. Bassoli, A. Gatto, L. Luliano, "Joining mechanisms and mechanical properties of PA composites obtained by selective laser sintering,” Rapid Prototyping Journal, 18 (2), 100-108 (2012).

[10] Roadmap for Additive Manufacturing: identify the future of freeform manufacturing, NSF workshop, University of Texas, (2009).

[11] Papadakis, L., Loizou, A., Risse, J., Bremen, S., \& Schrage, J. “A computational reduction model for appraising structural effects in selective laser melting manufacturing: a methodical model reduction proposed for time-efficient finite element analysis of larger components in Selective Laser Melting," Virtual and Physical Prototyping, 9(1), 17-25 (2014).

[12] Li, R., Liu, J., Shi, Y., Wang, L., \& Jiang, W., "Balling behavior of stainless steel and nickel powder during selective laser melting process." The International Journal of Advanced Manufacturing Technology, 59(9-12), 10251035 (2012).

[13] Hussein, A., Hao, L., Yan, C., \& Everson, R., "Finite element simulation of the temperature and stress fields in single layers built without-support in selective laser melting," Materials \& Design, 52, 638-647 (2013).

[14] Roberts, I. A., Wang, C. J., Esterlein, R., Stanford, M., \& Mynors, D. J., “A three dimensional finite element analysis of the temperature field during laser melting of metal powders in additive layer manufacturing," International Journal of Machine Tools and Manufacture, 49(12), 916-923 (2009).

[15] Hodge, N. E., Ferencz, R. M., \& Solberg, J. M., "Implementation of a thermomechanical model for the simulation of selective laser melting," Computational Mechanics, 54(1), 33-51 (2014).

[16] S. S. Sih, J. W. Barlow, "Measurement and prediction of the thermal conductivity of powders at high temperature", Solid Freeform fabrication symposium, Univ. of Texas, 321-329 (1994).

[17] Spears, Thomas, and Scott Gold. "In-process Sensing in Selective Laser Melting (SLM) Additive Manufacturing." Integrating Materials and Manufacturing Innovation, 5(1), 1-25 (2016).

[18] Gusarov, A.V, I Yadroitsev, Ph Bertrand, and I Smurov. "Model of Radiation and Heat Transfer in Laser-powder Interaction Zone at Selective Laser Melting." Journal of Heat Transfer, 131(7), 072101-1-072101-10 (2009).

[19] M. Tang, P.C. Pistorius, J. Beuth, "Geometric model to predict porosity of part produced in powder bed system," Materials Science and Technology (MS\&T), 129-135 (2015).

[20] Khairallah, Saad, and Andy Anderson. "Mesoscopic Simulation Model of Selective Laser Melting of Stainless Steel Powder," Journal of Materials Processing Tech, 214(11), 2627-2636 (2014).

[21] Masmoudi, Amal, Rodolphe Bolot, and Christian Coddet. "Investigation of the Laser-powder-atmosphere Interaction Zone During the Selective Laser Melting Process," Journal of Materials Processing Tech, 225, $122-132$ (2015). 
[22] Matsumoto, M, M Shiomi, K Osakada, and F Abe. "Finite Element Analysis of Single Layer Forming on Metallic Powder Bed in Rapid Prototyping by Selective Laser Processing," International Journal of Machine Tools and Manufacture, 42(1), 61-67 (2002).

[23] Ganeriwala, Rishi, and Tarek Zohdi. "Multiphysics Modeling and Simulation of Selective Laser Sintering Manufacturing Processes," Procedia CIRP, 14, 299-304 (2014).

[24] Li, C, C.H Fu, Y.B Guo, and F.Z Fang. "A Multiscale Modeling Approach for Fast Prediction of Part Distortion in Selective Laser Melting." Journal of Materials Processing Tech, 229 703-712 (2016).

[25] Y. Zhang, A. Faghri, "Melting of a subcooled mixed powder bed with constant heat flux heating," International Journal of Heat and Mass Transfer, 42, 775-788 (1999).

[26] S. Kolossov, et. al., "3D FE simulation for temperature evolution in the selective laser sintering process," International Journal of Machine Tools and Manufacture, 44(2), 117-123 (2003).

[27] H. Chung, S. Das, "Numerical modeling of scanning laser-induced melting, vaporization and resolidification in metals subjected to step heat flux input," International Journal of Heat and Mass Transfer, 47, 4153-4164 (2004).

[28] E.O. Olakanmi, R.F. Cochrane, K.W. Dalgarno, "Densification mechanism and microstructural evolution in selective laser sintering of Al-12Si powders," Journal of Materials Processing Technology, 211, 113-121 (2011). [29] J. P. Kruth, et. al, "Lasers and materials in selective laser sintering," Assembly Automation, 23,4, 2003.

[30] J.P. Kruth, G. Levy, F. Klocke, T.H.C. Childs, "Consolidation phenomena in laser and powder-bed based layered manufacturing," Annals of the CIRP 56(2), 703-759 (2007).

[31] K. Senthilkumaran et al. "Influence of building strategies on the accuracy of parts in selective laser sintering," Materials and Design, 30, 2946-2954 (2009).

[32] R. Paul, S. Anand, "Process energy analysis and optimization in selective laser sintering," Journal of Manufacturing Systems, 31, 429- 437 (2012).

[33] A.V. Ragulya, "Selective laser sintering, principles, continual model," Powder Metallurgy and Metal Ceramics, 37 (7-8), 356-364 (1998).

[34] A. Simchi, "Direct laser sintering of metal powders: Mechanism, kinetics and microstructural features," Materials Science and Engineering A, 428, 148-158 (2006).

[35] K. Dai, L. Shaw, "Thermal and mechanical finite element modeling of laser forming from metal and ceramic powders," Acta Materialia, 52(1), 69-80 (2003).

[36] A. Simchi, "The Role of Particle Size on the Laser Sintering of Iron Powder," Metallurgical and Materials Transactions B, 35B, 937-938 (2004).

[37] Wang, Xuan, Matthew Wraith, Stephen Burke, Howard Rathbun, and Kyle Devlugt. "Densification of W-Ni-Fe Powders Using Laser Sintering," International Journal of Refractory Metals and Hard Materials, 56, 145-150 (2016). [38] Olevsky, Eugene. "Theory of Sintering: From Discrete to Continuum," Materials Science \& Engineering R, 23(2), 41-100 (1998). 